\title{
Comparison of the Immediate Effect of the Whole-body Vibration on Proprioceptive Precision of the Knee Joint Between Barefoot and Shoe-wearing Conditions in Healthy Participants
}

\author{
Yu-bin Lee ${ }^{1}$, BPT, PT, Ui-jae Hwang ${ }^{2,3}$, PhD, PT, Oh-yun Kwon²,3, PhD, PT \\ ${ }^{1}$ Department of Physical Therapy, The Graduate School, Yonsei University, ${ }^{2}$ Department of Physical Therapy, College of Health Science, \\ Yonsei University, ${ }^{3}$ Kinetic Ergocise Based on Movement Analysis Laboratory, Wonju, Korea
}

\author{
Article Info \\ Received April 12, 2021 \\ Revised April 18, 2021 \\ Accepted April 20, 2021 \\ Corresponding Author \\ Oh-yun Kwon \\ E-mail: kwonoy@yonsei.ac.kr \\ https://orcid.org/0000-0002-9699-768X
}

\section{Key Words}

Proprioception

Shoes

Vibration
Background: Whole-body vibration (WBV) has been used to alleviate proprioceptive damage by musculoskeletal and neurological conditions. However, no study has determined whether wearing shoes while applying WBV can affect proprioception precision of the knee joint.

Objects: This study aimed to determine the differences in the proprioceptive precision of the knee joint before and after WBV and to compare the proprioceptive precision of the knee joint between barefoot and shoe-wearing conditions.

Methods: This study recruited 33 healthy participants. A passive-to-active angle reproduction test was used to measure the proprioception precision of the knee joint using an electrogoniometer, and the target angle was set to a knee flexion of $30^{\circ}$. Proprioception precision was calculated using the error angle (angular difference from $30^{\circ}$ ). Proprioceptive precision was measured in weight-bearing and non-weight-bearing positions before and after applying WBV for 20 minutes at $12 \mathrm{~Hz}$ in barefoot and shoe-wearing conditions. Mixed repeated analysis of variance was used to determine the differences in changes in the proprioceptive precision of the knee joint according to foot conditions.

Results: There were significant improvements in the weight-bearing ( $p=0.002)$ and nonweight-bearing $(p<0.001)$ proprioceptive precision of the knee joint after applying WBV. However, there was no significant difference in the change in proprioceptive precision of the knee joint after applying WBV between the barefoot and shoe-wearing conditions.

Conclusion: WBV stimulation had an immediate effect on improving the proprioceptive precision of the knee joint. However, foot conditions (barefoot or shoe-wearing) during WBV application did not influence the proprioceptive precision of the knee joint.

\section{INTRODUCTION}

To maintain postural control, it is necessary to detect body movements through the sensory system, integrate sensorimotor information within the central nervous system, and execute motor responses appropriately [1]. To sense the movement of the body, the visual, vestibular, and somatosensory systems are essential $[2,3]$. Peripheral receptors of the somatosensory system consist of muscle spindles, Golgi tendon organs, joint receptors, and cutaneous receptors [4,5]. These receptors are embedded in the muscle fibers, tendons, and joint capsules [4,5]. Patients with neurological impairments show abnormal postural control due to an inability to adapt to sensory input on account of pathological disruptions within the sensory system or damage to the central sensorimotor structure, which is important for organizing sensory information [6]. Patients with musculoskeletal pathologies, including low back pain, ankle sprain, and degenerative joint disease, present with impairment of proprioceptive sensations [7,8].

Several proprioception and balance training methods (such as balance board and uneven surface training) have been applied and demonstrated to improve sensorimotor adaptation and proprioception [1]. Recently, whole-body vibration (WBV) has been applied to improve proprioception as well as balance, strength, and power in various fields such as sports, geriatric wellness, rehabilitation, and fitness [9-12]. WBV can stim- 
ulate the joints, tendons, and muscles simultaneously through low-frequency vibrations generated by a specialized machine [9,13-15]. Previous studies have reported that WBV was risk factor for some musculoskeletal disorders such as lower back pain but it was effective in improving proprioception $[9,12,16$. However, there have been no consistent results regarding the effects of WBV on proprioception precision improvement [17]. Some studies have reported that the sole of a shoe increases the surface area of the foot that is in contact with a vibrating surface, thus increasing vibration stimulation [18,19]. In contrast, another study reported that the flexibility of the shoe sole can reduce the amplitude and frequency of vibrations and that more vibrations can be transmitted to the ankle under barefoot conditions [19]. As such, the effect of vibrations according to footwear conditions is controversial. Accordingly, the present study was aimed at comparing the effects of WBV stimulation on proprioceptive precision of the knee joint between barefoot and shoe-wearing conditions. Previous studies have typically investigated proprioceptive precision of the knee joint on an open kinetic chain (non-weight-bearing position) after WBV stimulation [9,20-22]. WBV stimulation is usually performed in a closed chain position (weight-bearing position) on a plate, whereas standing balance tests are performed in the weightbearing position. However, no study has determined whether WBV stimulation can improve the proprioceptive precision of the knee joint in the weight-bearing position. Determining whether WBV stimulation can improve the proprioceptive precision of the knee joint in both non-weight-bearing and weight-bearing positions and evaluating the differences in the effects of WBV stimulation between shoe-wearing and barefoot conditions would provide valuable information for retraining proprioception and balance rehabilitation. Thus, the purpose of this study was to determine the proprioception precision of the knee joint before and after applying WBV and to compare the effects of WBV stimulation on proprioception precision of the knee joint between foot conditions (wearing shoes vs. barefoot). We hypothesized that proprioception precision of the knee joint would be different between foot conditions and that the barefoot condition would be better effect of WBV than the shoes condition. And hypothesized that proprioception precision of the knee joint would be different between weight bearing conditions.

\section{MATERIALS AND METHODS}

\section{Participants}

Although 33 participants were originally recruited for this study, only 30 participants ultimately completed the study. Three participants dropped out for personal reasons. The demographic data are shown in Table 1. This research protocol was reviewed and approved by the Yonsei University Mirae Institutional Review Board (approval number 1041849-202101BM-001-02). All participants provided written consent before participating in the study. Healthy participants without any musculoskeletal or neurological conditions were recruited. The exclusion criteria were as follows: (1) history of fracture or surgery on the lower extremities; (2) lower extremity injury; (3) sensory deficits and musculoskeletal dysfunction or neurological impairment; and (4) discomfort or dizziness during delivery of the vibratory stimulus used in this study.

\section{Proprioception Precision Test of the Knee Joint}

The passive-to-active angle reproduction test was used to measure the proprioception precision of the knee joint. A digital goniometer (iGAGING, Los Angeles, CA, USA) was used to measure the knee joint angle. The intra-rater reliability of knee joint measurement using a digital goniometer was 0.997-0.998, and the inter-rater reliability was 0.994 [23]. A digital goniometer was placed on the lateral part of the femur and leg parallel to the line that links the great trochanter and the lateral epicondyle of the femur and lateral malleolus of the fibula. The axis of the digital goniometer was located at the center of the knee joint [24]. The dominant leg, determined by kicking ball performance, was selected as the testing side [24].

Table 1. The characteristics of the subjects

\begin{tabular}{lcccc}
\hline \multicolumn{1}{c}{ Group } & Age $(\mathrm{y})$ & Body mass index $\left(\mathrm{kg} / \mathrm{m}^{2}\right)$ & Height $(\mathrm{cm})$ & Weight $(\mathrm{kg})$ \\
\hline All $(\mathrm{N}=30)$ & $28.0 \pm 2.13$ & $19.3 \pm 2.83$ & $169.2 \pm 8.66$ & $65.5 \pm 11.94$ \\
Male $(\mathrm{n}=20)$ & $28.6 \pm 2.19$ & $20.6 \pm 2.17$ & $173.9 \pm 5.49$ & $71.7 \pm 8.42$ \\
Female $(\mathrm{n}=10)$ & $27.1 \pm 1.73$ & $16.6 \pm 2.07$ & $159.6 \pm 5.06$ & $53.2 \pm 7.52$ \\
\hline
\end{tabular}

Values are presented as mean \pm standard deviation. 
To measure the proprioceptive precision of the knee joint, the principal investigator (PI) moved the participant's leg passively at a target knee angle of $30^{\circ}$ flexion and stopped, and the participant was given 4 seconds to estimate the knee angle [25]. Then, the PI replaced the leg passively into the starting position, and the participant was asked to actively move the tested leg to position the knee joint at the previously estimated angle to reproduce it. When the participant reported that they had reproduced the knee joint to exactly the remembered position, the PI measured this angle with a digital goniometer. Proprioception precision was calculated using the error angle (angular difference from $30^{\circ}$ ). Three trials were performed, and the average value of the three trials was calculated and used for data analysis. This measurement was performed at two different positions (weight-bearing and non-weight-bearing positions) and in two different foot conditions (barefoot and while wearing shoes). The order in which the measurements were taken was selected randomly.

\section{1) Weight-bearing position test (WB)}

In the weight-bearing position test (WB), the participants were barefoot and clothed to above the middle of their thighs prior to measurement in order to abolish cutaneous sensations. Participants stood next to a table, closed their eyes, and lightly touched the edge of a table to maintain balance during the test. The participants were then asked to lift the opposite foot of the tested leg [25].

The PI passively moved the knee joint of the tested leg to a target knee angle of $30^{\circ}$ stopped with verbal cue and contact. The participant was asked to estimate the knee angle during 4 seconds [25]. Then, the PI replaced the leg passively into the starting position, and the participant was asked to actively flex the knee joint at the remembered positional angle to reproduce it. When the participant reported that they had reproduced the knee joint to exactly the remembered position, the PI measured the knee angle (Figure 1). Three trials were performed, and the average value of the three trials was calculated.
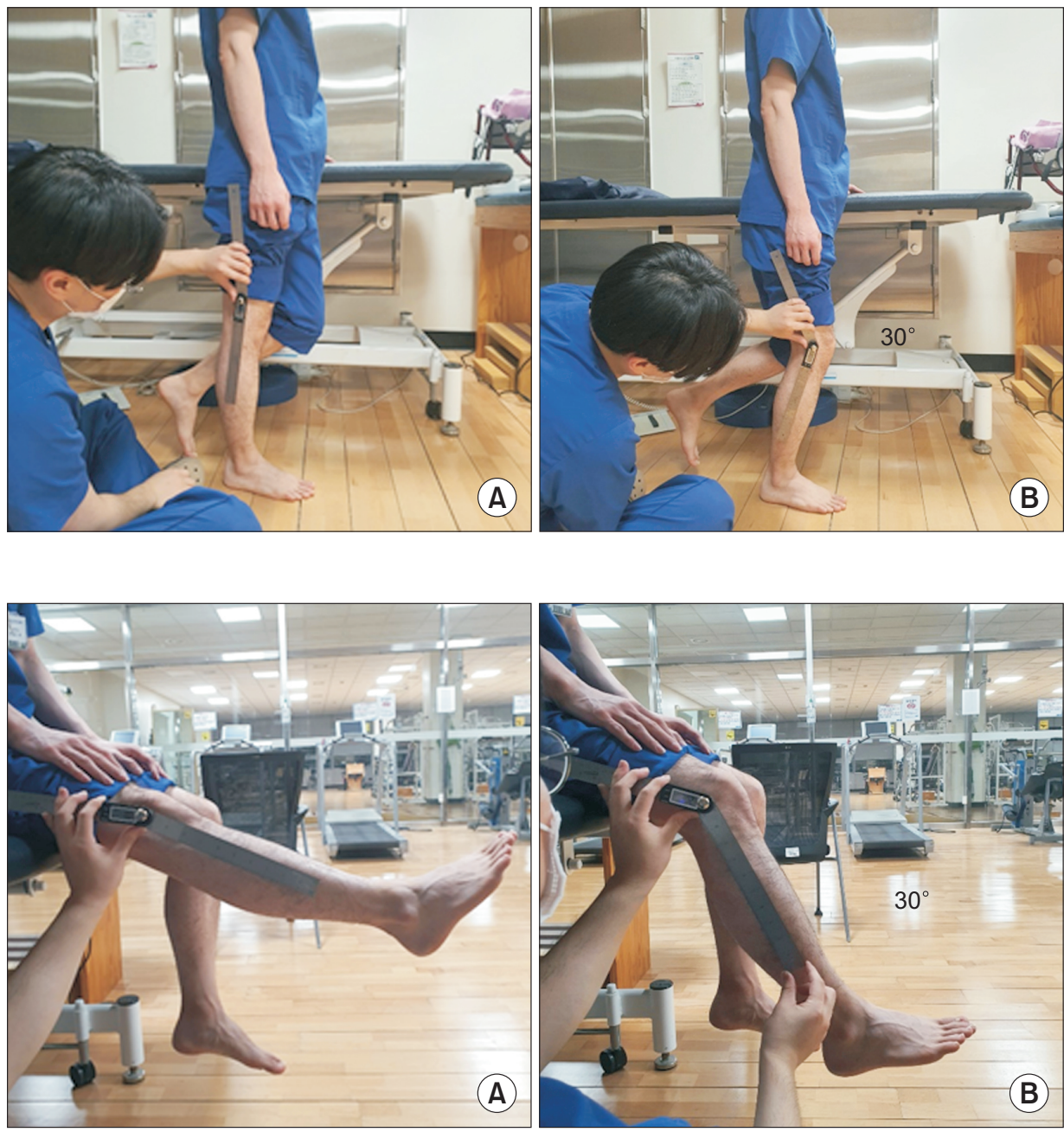

Figure 1. (A) Measuring the precision of proprioception of the knee joint in weight bearing position (start position). (B) Measuring the precision of proprioception of the knee joint in weight bearing position (end position).
Figure 2. (A) Measuring the precision of proprioception of the knee joint in nonweight bearing position (start position). (B) Measuring the precision of proprioception of the knee joint in non-weight bearing position (end position). 


\section{2) Non-weight bearing position test (NWB)}

In the NWB, the participants sat on a table barefoot and did not touch the floor with their feet before measurement. The participants were then asked to close their eyes during measurements. The proprioceptive precision measurement in the non-weight-bearing position was performed as in the WB procedures (Figure 2).

\section{Whole-Body Vibration (WBV)}

The Galileo ${ }^{\circledR}$ Delta A Tilt Table (Novotec Medical GmbH, Pforzheim, Germany) was used for WBV stimulation. WBV stimulation was applied for 20 minutes and at $12 \mathrm{~Hz}$ in accordance with the manufacturer's website. Participants stood with their legs spread apart to shoulder width and looked straight ahead (Figure 3). Within 5 minutes after intervention, proprioception precision tests with WB and NWB were conducted randomly. WBV stimulation and measurement of proprioceptive precision of the knee joint for each foot condition were performed weeks apart in order to minimize learning effects. Participants wore indoor shoes for the shoe-wearing condition. The test order was randomly selected.

\section{Statistical Analysis}

All statistical analyses were performed using the SPSS software (ver. 25.0 for Windows; IBM Corp., Armonk, NY, USA). The average and standard deviation were calculated for all variables. The Shapiro-Wilk test was used to test for normality. A $2 \times 2$ (foot conditions [barefoot, wearing shoes] $\times$ time [pre, post]) repeated-measures analysis of variance was per- formed to assess within and between differences in the precision of proprioception of the knee joint. An independent $t-$ test was performed to compare the proprioception precision of the knee joint between WB and NWB. A paired t-test was performed to compare the precision of proprioception of the knee joint pre- and post-WBV. The level of statistical significance was set at $5 \%(\mathrm{p}<0.05)$.

\section{RESULTS}

All variables were normally distributed. There was a significant main effect in the precision of proprioception of the knee joint between pre- and post-WBV in WB ( $\mathrm{p}=0.002)($ Table 2$)$

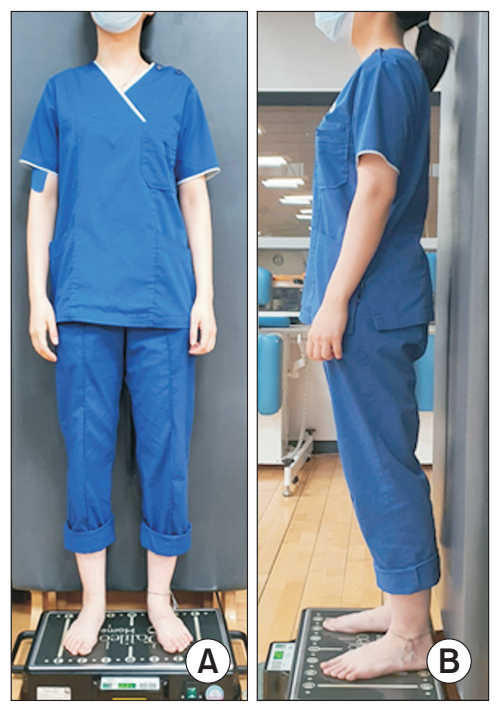

Figure 3. (A) Whole body vibration stimulation position (front). (B) Whole body vibration stimulation position (side).

Table 2. Within (pre- and post-WBV) and between (barefoot and wearing shoes) differences in precision of proprioception of the knee joint measured in WB position

\begin{tabular}{|c|c|c|c|c|c|c|}
\hline \multirow{2}{*}{ Variable } & \multirow{2}{*}{$\mathrm{FC}$} & \multicolumn{2}{|c|}{ Proprioception } & \multirow{2}{*}{$\begin{array}{l}\text { Interaction effect } \\
\text { FC } \times \text { WBV }(p)\end{array}$} & \multirow{2}{*}{$\begin{array}{l}\text { Significant difference } \\
\text { within WBV (p) }\end{array}$} & \multirow{2}{*}{$\begin{array}{l}\text { Significant difference } \\
\text { between FC (p) }\end{array}$} \\
\hline & & Pre & Post & & & \\
\hline \multirow[t]{2}{*}{ WB } & Barefoot & $2.55 \pm 1.72$ & $1.48 \pm 1.38$ & 0.751 & $0.002^{*}$ & 0.964 \\
\hline & Shoes & $2.45 \pm 1.77$ & $1.55 \pm 1.71$ & & & \\
\hline
\end{tabular}

Values are presented as mean \pm standard deviation. FC, foot condition; WBV, whole body vibration; WB, weight bearing. ${ }^{*}$ Significant difference (p < 0.05$)$.

Table 3. Within (pre- and post-WBV) and between (barefoot and wearing shoes) differences in precision of proprioception of the knee joint measured in NWB position

\begin{tabular}{|c|c|c|c|c|c|c|}
\hline \multirow{2}{*}{ Variable } & \multirow{2}{*}{$\mathrm{FC}$} & \multicolumn{2}{|c|}{ Proprioception } & \multirow{2}{*}{$\begin{array}{l}\text { Interaction effect } \\
\text { FC } \times \text { WBV }(p)\end{array}$} & \multirow{2}{*}{$\begin{array}{c}\text { Significant difference } \\
\text { within WBV (p) }\end{array}$} & \multirow{2}{*}{$\begin{array}{c}\text { Significant difference } \\
\text { between FC (p) }\end{array}$} \\
\hline & & Pre & Post & & & \\
\hline \multirow[t]{2}{*}{ NWB } & Barefoot & $3.64 \pm 2.39$ & $2.55 \pm 2.37$ & 0.175 & $<0.001 *$ & 0.656 \\
\hline & Shoes & $3.85 \pm 2.37$ & $1.91 \pm 1.50$ & & & \\
\hline
\end{tabular}

Values are presented as mean \pm standard deviation. FC, foot condition; WBV, whole body vibration; NWB, non-weight bearing. *Significant difference lp < 0.05). 
and NWB positions ( $<$ 0.001) (Table 3). Paired t-tests showed significant differences in the precision of proprioception of the knee joint between pre-and post-WBV in both barefoot ( $p$ $=0.014)$ and shoe-wearing conditions $(\mathrm{p}=0.015)$ in the WB position, and barefoot $(p=0.025)$ and shoe-wearing conditions $(\mathrm{p}<0.001)$ in the NWB position (Figure 4). Pre-WBV, independent t-tests showed significant differences in the precision of proprioception of the knee joint between WB and NWB positions in both barefoot $(p=0.046)$ and shoe-wearing conditions $(\mathrm{p}=0.012)$ (Table 4). Post-WBV, both barefoot $(\mathrm{p}=$ $0.038)$ and shoe-wearing conditions ( $p=0.026)$ showed significant differences in the precision of proprioception of the knee joint between the WB and NWB positions (Table 5). However, there were no significant main effects or interactive effects on the precision of proprioception of the knee joint between foot conditions in WB ( $\mathrm{p}=0.964)$ (Table 2$)$ and NWB positions ( $\mathrm{p}=$ 0.656) (Table 3).

\section{DISCUSSION}

This study was conducted to determine the effects of WBV stimulation on proprioceptive precision of the knee joint measured in the NWB and WB positions and to compare the effects of WBV stimulation on proprioceptive precision of the knee joint between barefoot and shoe-wearing conditions. Our hypothesis was that proprioception precision of the knee joint
A



Non-weight bearing

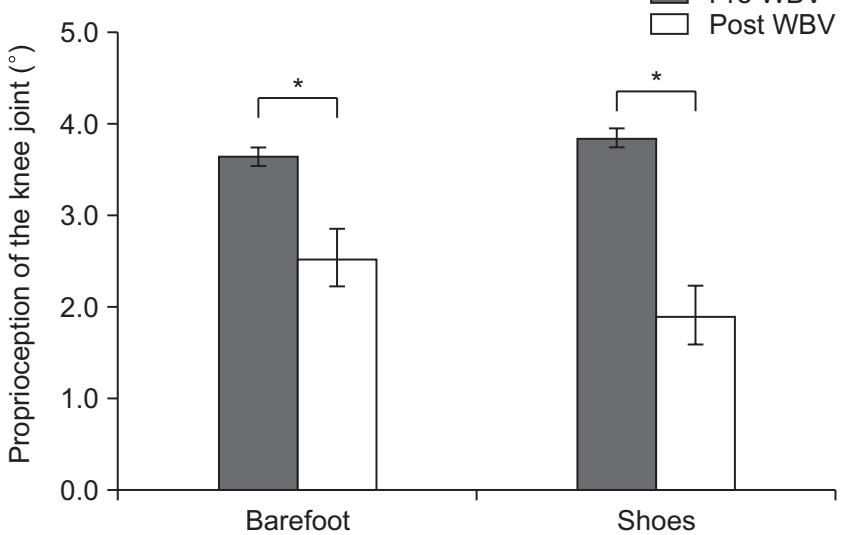

Figure 4. (A) Comparison of precision of proprioception of the knee joint between pre and post-WBV (weight bearing). (B) Comparison of precision of proprioception of the knee joint between pre and post-WBV (non-weight bearing). WBV, whole body vibration.

Table 4. Comparison of precision of proprioception of the knee joint measured pre-WBV between WB and NWB test position

\begin{tabular}{|c|c|c|c|c|c|c|}
\hline \multirow{2}{*}{ Foot conditions } & \multirow{2}{*}{$\begin{array}{c}\text { Test } \\
\text { position }\end{array}$} & \multicolumn{3}{|c|}{ Proprioception } & \multirow{2}{*}{$\mathrm{t}$} & \multirow{2}{*}{$\mathrm{p}$} \\
\hline & & $\mathrm{N}$ & Pre-mean & SD & & \\
\hline \multirow[t]{2}{*}{ Barefoot } & WB & 30 & 2.55 & 1.72 & 2.038 & $0.046 *$ \\
\hline & NWB & 30 & 3.64 & 2.39 & & \\
\hline \multirow[t]{2}{*}{ Shoes } & WB & 30 & 2.45 & 1.77 & 2.596 & $0.012^{*}$ \\
\hline & NWB & 30 & 3.85 & 2.37 & & \\
\hline
\end{tabular}

SD, standard deviation; WB, weight bearing; NWB, non-weight bearing. *Significant difference $(p<0.05)$.

Table 5. Comparison of precision of proprioception of the knee joint measured post WBV between WB and NWB test position

\begin{tabular}{|c|c|c|c|c|c|c|}
\hline \multirow{2}{*}{ Foot conditions } & \multirow{2}{*}{$\begin{array}{c}\text { Test } \\
\text { position }\end{array}$} & \multicolumn{3}{|c|}{ Proprioception } & \multirow{2}{*}{$t$} & \multirow{2}{*}{$\mathrm{p}$} \\
\hline & & $\mathrm{N}$ & Post-mean & SD & & \\
\hline \multirow[t]{2}{*}{ Barefoot } & WB & 30 & 1.48 & 1.38 & 2.126 & $0.038^{*}$ \\
\hline & NWB & 30 & 2.55 & 2.37 & & \\
\hline \multirow[t]{2}{*}{ Shoes } & WB & 30 & 1.12 & 1.16 & 2.284 & $0.026^{*}$ \\
\hline & NWB & 30 & 1.91 & 1.50 & & \\
\hline
\end{tabular}

SD, standard deviation; WB, weight bearing; NWB, non-weight bearing. *Significant difference $(p<0.05)$. 
would be differences according to foot conditions and that there would be differences according to weight bearing conditions. The results of the study showed that WBV was effective for the proprioception of the knee joint, but there were no significant differences between foot conditions. Several studies have demonstrated that proprioception of the knee joint improves after WBV stimulation [10,13,26-28].

Lin et al. [27] reported that the error angle of knee joint proprioception decreased by approximately $1.5^{\circ}-1.9^{\circ}$ after applying WBV in healthy adult males. Zahedi et al. [28] reported that the error angle of knee joint proprioception decreased by $0.8^{\circ}-1.2^{\circ}$ in healthy adults and $0.7^{\circ}$ in patients with osteoarthritis. Furthermore, Ko et al. [26] studied the effect of WBV stimulation in children with cerebral palsy and reported that the error angle of knee joint proprioception decreased by $1.28^{\circ}$ after WBV stimulation. Similarly, the results of the present study showed that the error angle of knee joint proprioception after applying WBV decreased by $1.07^{\circ}$ in the barefoot condition and by $0.9^{\circ}$ in the shoe-wearing condition. These results support the hypothesis that WBV can improve proprioception precision of the knee joint.

The results of the present study showed that proprioception precision of the knee joint significantly improved after WBV stimulation in both foot conditions. However, there is a lack of scientific evidence of the effect of WBV on improving proprioception of the knee joint. A possible mechanism by which proprioception precision of the knee joint was significantly improved after WBV stimulation is explained below.

Mense [29] studied the effect of temperature on muscle spindles and tendon organs and reported that muscle warming increased the firing rate of group Ia afferents and the Golgi tendon organs. Cochrane et al. [30] studied the effect of acute WBV stimulation on muscle temperature and found that the mean rate of increase in muscle temperature was significantly increased during acute WBV and that the rate of muscle temperature during acute WBV was approximately twice that achieved by cycle and hot water immersion interventions. WBV can evoke friction between soft tissues during WBV application, which may increase the muscle temperature. In addition, WBV may lead to rhythmic muscle contraction, which increases muscle temperature via active leg muscle contraction and increased metabolism of the major muscles. Although temperatures of the surrounding knee joint muscles were not measured directly in the present study, increased muscle tem- perature may increase the firing rate of group Ia afferents and Golgi tendon organs, thereby contributing to improvements in movement detection threshold and the precision of proprioception of the knee joint after WBV delivery.

Previous studies have reported that WBV exposure has adverse effects on proprioceptive precision and sensory acuity [31,32]. However, some studies have reported that proprioceptive precision did not change after WBV stimulation [19,33]. Hannah et al. [17], Pollock et al. [19], and Jones et al. [34] reported that WBV did not affect proprioceptive precision of the knee joint. In the present study, the precision of proprioception of the knee joint significantly improved after 20 minutes of WBV stimulation. These inconsistencies between the results of previous studies may be explained by differences in the type of vibrators and parameters of the vibratory stimuli used during these studies. Further studies are needed to confirm whether proprioceptive precision can be influenced by different parameters such as stimulation time, frequency, position during vibration stimulation, pattern of stimulation, type of vibratory machine, and magnitude of vibration.

Wearing shoes can affect balance, sensory input, and proprioception function. The cushion and type of sole of the shoes can influence the amplitude and frequency of the vibration. The amplitude of vibration can be reduced while standing by wearing shoes with flexible soles, and the amount of transmitted vibration can be enhanced by increasing the surface area of the foot compared with being barefoot [18,20]. Threshold values of the vibration are different between barefoot and shoe-wearing conditions, and the threshold value while being barefoot $(31.1 \mu \mathrm{m})$ was lower than that while wearing shoes $(42.1 \mu \mathrm{m})$. Being barefoot has a higher vibration sensitivity [35]. Therefore, the current study hypothesized that the effect of WBV stimulation on proprioception precision of the knee joint would be different between barefoot and shoe-wearing conditions during WBV application. The results of the current study showed that there was no significant difference in the effect of WBV stimulation between barefoot and shoe-wearing conditions. In this study, indoor shoes were used to apply the WBV. The soles of indoor shoes are relatively thin and flexible compared to sports shoes. The relatively thin and flexible sole of indoor shoes may have contributed to the results of this study. Further studies are needed to determine the effect of WBV stimulation on the proprioception precision of the knee joint using sports shoes with thick and stiff soles. Determining 
whether wearing shoes during WBV shows proprioception precision of the knee joint that is as effective as that in barefoot conditions would provide valuable clinical information.

Proprioception of the knee joint can be influenced by different weight-bearing conditions (closed kinetic chain vs. open kinetic chain) $[24,25,36]$. Previous studies have reported that proprioception in the knee of the dominant leg measured on WB showed a smaller error value of approximately $2^{\circ}-3^{\circ}$ than that on NWB, resulting in more accurate and functionally relevant results [24,35-37]. The results of the current study also showed significantly less precision about $1.1^{\circ}$ in the proprioception of the knee joint in the WB position than in the NWB position. The proprioception test on WB was more strongly influenced by the resistance of the lower extremity muscles than on NWB. In addition, cutaneous sensory receptors of the sole of the foot, mechanoreceptors in the hip and ankle joints and muscle spindles of the hip and ankle muscles are stimulated simultaneously during the proprioception test of the knee joint on WB. Because of these effects, it is difficult to isolate knee joint movements from sensory input of the hip and ankle joints and muscles [24,25,38-40]. Conversely, NWB does not assess movements, resistance, or sensory input from adjacent joints, so it is likely that the NWB exclusively considers movements of the knee joint. Especially in the present study, the WB condition would be affected by eccentric contraction of quadriceps muscle and the NWB condition would have affected the knee flexion by hamstring muscle. When proprioception precision test of the knee joint, all of these factors that can affect the test results, such as verbal cue, body weight, muscle strength and touch support when unilateral balance control, should be considered.

Prolonged exposure to vibrations has been shown to have detrimental effects on soft tissues, which include muscle fatigue, reductions in motor unit firing rates and muscle contraction force, decreases in nerve conduction velocity, and attenuated perception $[41,42]$. Further studies are required to determine the optimal stimulation time and frequency.

This study had some limitations. First, the tester was not blinded from test condition which would interfere with the experiment. Second, each participant had different parts to add weights to. As a result, we could not obtain accurate results because different parts were stimulated by the vibration, and the degree of stimulation varied depending on the angle of the knee [43]. Third, the measurements were performed only at an angle of $30^{\circ}$. This makes it difficult to generalize the results of this study to different angles. For these reasons, it is necessary to evaluate several participants using various angles, taking into account the precise posture for proprioception stimulation of the knee.

\section{CONCLUSIONS}

We compared the differences in the proprioceptive precision of the knee joint after WBV according to foot conditions. In conclusion, WBV was effective in improving proprioception of the knee, regardless of whether the participants wore footwear. The results of the current study indicate that WBV stimulation is effective for improving the precision of proprioception of the knee joint in both WB and NWB testing positions.

\section{CONFLICTS OF INTEREST}

No potential conflict of interest relevant to this article was reported.

\section{AUTHOR CONTRIBUTIONS}

Conceptualization: YL. Data curation: YL. Formal analysis: UH, OK. Investigation: YL. Methodology: YL, UH, OK. Project administration: UH, OK. Resources: YL, UH, OK. Supervision: YL, UH, OK. Visualization: YL, UH. Writing - original draft: YL, UH, OK. Writing - review \& editing: YL, UH, OK.

\section{ORCID}

Yu-bin Lee, https://orcid.org/0000-0002-6876-6331

Ui-jae Hwang, https://orcid.org/0000-0002-2050-5503

\section{REFERENCES}

1. Hammer A, Nilsagård Y, Wallquist M. Balance training in stroke patients - a systematic review of randomized, controlled trials. Adv Physiother 2008;10(4):163-72.

2. Patel M, Gomez S, Lush D, Fransson PA. Adaptation and vision change the relationship between muscle activity of the lower limbs and body movement during human balance perturbations. Clin Neurophysiol 2009;120(3):601-9.

3. Ruhe A, Fejer R, Walker B. The test-retest reliability of centre 
of pressure measures in bipedal static task conditions--a systematic review of the literature. Gait Posture 2010;32(4):43645.

4. Solomonow M, Krogsgaard M. Sensorimotor control of knee stability. A review. Scand J Med Sci Sports 2001;11(2):64-80.

5. Riemann BL, Lephart SM. The sensorimotor system, part II: the role of proprioception in motor control and functional joint stability. J Athl Train 2002;37(1):80-4.

6. Bowerman CC, Semrau JA, Kiss Z Dukelow SP. The importance of somatosensory deficits in neurological disease. Paper presented at: 17th Annual International Functional Electrical Stimulation Society (IFESS); 2012 Sep 9-12; Banff, Canada. Graz: IFESS, 2012. pp. 2-5.

7. Knoop J, Steultjens MP, van der Leeden M, van der Esch M, Thorstensson CA, Roorda LD, et al. Proprioception in knee osteoarthritis: a narrative review. Osteoarthritis Cartilage 2011;19(4):381-8.

8. Clark NC, Röijezon U, Treleaven J. Proprioception in musculoskeletal rehabilitation. Part 2: clinical assessment and intervention. Man Ther 2015;20(3):378-87.

9. Hopkins T, Pak JO, Robertshaw AE, Feland JB, Hunter I, Gage M. Whole body vibration and dynamic restraint. Int J Sports Med 2008;29(5):424-8.

10. Moezy A, Olyaei G, Hadian M, Razi M, Faghihzadeh S. A comparative study of whole body vibration training and conventional training on knee proprioception and postural stability after anterior cruciate ligament reconstruction. Br J Sports Med 2008;42(5):373-8.

11. Trans T, Aaboe J, Henriksen M, Christensen R, Bliddal H, Lund H. Effect of whole body vibration exercise on muscle strength and proprioception in females with knee osteoarthritis. Knee 2009;16(4):256-61.

12. Cormie P, Deane RS, Triplett NT, McBride JM. Acute effects of whole-body vibration on muscle activity, strength, and power. J Strength Cond Res 2006;20(2):257-61.

13. Eklund G. On muscle vibration in man; an amplitude-dependent inhibition, inversely related to muscle length. Acta Physiol Scand 1971;83(3):425-6.

14. Eklund G. Position sense and state of contraction; the effects of vibration. J Neurol Neurosurg Psychiatry 1972;35(5):60611.

15. Burke D, Hagbarth KE, Löfstedt L, Wallin BG. The responses of human muscle spindle endings to vibration of non-contracting muscles. J Physiol 1976;261(3):673-93.
16. Brooke-Wavell K, Mansfield NJ. Risks and benefits of whole body vibration training in older people. Age Ageing 2009;38(3):254-5.

17. Hannah R, Minshull C, Folland JP. Whole-body vibration does not influence knee joint neuromuscular function or proprioception. Scand J Med Sci Sports 2013;23(1):96-104.

18. Marín PJ, Bunker D, Rhea MR, Ayllón FN. Neuromuscular activity during whole-body vibration of different amplitudes and footwear conditions: implications for prescription of vibratory stimulation. J Strength Cond Res 2009;23(8):2311-6.

19. Pollock RD, Provan S, Martin FC, Newham DJ. The effects of whole body vibration on balance, joint position sense and cutaneous sensation. Eur J Appl Physiol 2011;111(12):306977.

20. Saade N. Whole-body vibration transmission barefoot and with shoes in athletes and sedentary individuals. Montreal, Concordia University, Master's Thesis. 2013.

21. Madill S. The immediate influence of whole body vibration on proprioceptive precision. Utah, Utah State University, Master's Thesis. 2018.

22. Goodwin GM, McCloskey DI, Matthews PB. Proprioceptive illusions induced by muscle vibration: contribution by muscle spindles to perception? Science 1972;175(4028):1382-4.

23. Svensson M, Lind V, Löfgren Harringe M. Measurement of knee joint range of motion with a digital goniometer: a reliability study. Physiother Res Int 2019;24(2):e1765.

24. Hanafy AF. Weight-bearing and non-weight bearing proprioception assessment of dominant and non-dominant lower limbs in adult females. JMSCR 2017;5(2):17484-92.

25. Lokhande MV, Shetye J, Mehta A, Deo MV. Assessment of knee joint proprioception in weight bearing and in non-weight bearing positions in normal subjects. JKIMSU 2013;2(2):94101.

26. Ko MS, Sim YJ, Kim DH, Jeon HS. Effects of three weeks of whole-body vibration training on joint-position sense, balance, and gait in children with cerebral palsy: a randomized controlled study. Physiother Can 2016;68(2):99-105.

27. Lin HT, Chen YY, Wang DC, Chou PH, Guo LY, Wu WL. The acute effect of training frequencies and number of sets of whole body vibration on knee joint proprioception. J Mech Med Biol 2014;14(3):1450036.

28. Zahedi A, Tajali SB, Oliaye G, Naemi SS, Kalantari KK. Effects of short-term whole body vibration training on knee proprioception in healthy adults and people with knee osteoarthritis. 
J Clin Physiother Res 2017;2(3):119-25.

29. Mense S. Effects of temperature on the discharges of muscle spindles and tendon organs. Pflugers Arch 1978;374(2):15966.

30. Cochrane DJ, Stannard SR, Sargeant AJ, Rittweger J. The rate of muscle temperature increase during acute whole-body vibration exercise. Eur J Appl Physiol 2008;103(4):441-8.

31. Cordo P, Gurfinkel VS, Bevan L, Kerr GK. Proprioceptive consequences of tendon vibration during movement. J Neurophysiol 1995;74(4):1675-88.

32. Oullier O, Kavounoudias A, Duclos C, Albert F, Roll JP, Roll R. Countering postural posteffects following prolonged exposure to whole-body vibration: a sensorimotor treatment. Eur J Appl Physiol 2009;105(2):235-45

33. Santos BR, Larivière C, Delisle A, Plamondon A, Boileau PÉ, Imbeau D. A laboratory study to quantify the biomechanical responses to whole-body vibration: the influence on balance, reflex response, muscular activity and fatigue. International Journal of Industrial Ergonomics 2008;38(7-8):626-39.

34. Jones A, Gordon S, Sealey R. The effect of a single session of whole body vibration on mobility, lower limb joint position sense and balance of community dwelling older adults. Internet J Allied Health Sci Pract 2013;11(3):Article 7.

35. Schlee G, Sterzing T, Milani TL. Influence of footwear on foot sensitivity: a comparison between barefoot and shod sports. Paper presented at: 25th International Symposium on Biomechanics in Sports (ISBS); 2007 Aug 23-27; Ouro Preto, Brazil. Konstanz: ISBS, 2007. pp. 285-8.
36. Drouin JM, Houglum PA, Perrin DH, Gansneder BM. Weightbearing and non-weight-bearing knee-joint reposition sense and functional performance. J Sport Rehabil 2003;12(1):5466.

37. Ghiasi F, Akbari A. Comparison of the effects of open and closed kinematic chain and different target position on the knee joint position sense. J Med Sci 2007;7(6):969-76.

38. Verschueren SM, Swinnen SP, Cordo PJ, Dounskaia NV. Proprioceptive control of multijoint movement: unimanual circle drawing. Exp Brain Res 1999;127(2):171-81.

39. Abelew TA, Miller MD, Cope TC, Nichols TR. Local loss of proprioception results in disruption of interjoint coordination during locomotion in the cat. J Neurophysiol 2000;84(5):2709-14.

40. Wilson LR, Gandevia SC, Burke D. Discharge of human muscle spindle afferents innervating ankle dorsiflexors during target isometric contractions. J Physiol 1997;504(Pt 1):221-32.

41. Stewart JA, Cochrane DJ, Morton RH. Differential effects of whole body vibration durations on knee extensor strength. J Sci Med Sport 2009;12(1):50-3.

42. Rittweger J, Beller G, Felsenberg D. Acute physiological effects of exhaustive whole-body vibration exercise in man. Clin Physiol 2000;20(2):134-42.

43. Ritzmann R, Gollhofer A, Kramer A. The influence of vibration type, frequency, body position and additional load on the neuromuscular activity during whole body vibration. Eur J Appl Physiol 2013;113(1):1-11. 